\title{
Prognostic significance of the combination of preoperative fibrinogen and the neutrophil-lymphocyte ratio in patients with non-small cell lung cancer following surgical resection
}

\author{
HUA-GANG LIANG, KUN GAO, RUI JIA, JIAN LI and CHAO WANG \\ Department of Thoracic Surgery, The First Hospital of Qinhuangdao, Qinhuangdao, Hebei 066000, P.R. China
}

Received November 29, 2017; Accepted March 26, 2018

DOI: $10.3892 / \mathrm{ol} .2018 .9760$

\begin{abstract}
The purpose of the present study was to evaluate the prognostic value of preoperative coagulation factor levels (including fibrinogen and D-dimer) and inflammatory indicators in patients with non-small cell lung cancer (NSCLC). The medical records of 456 patients with NSCLC who had undergone curative resection were retrospectively analysed. The recommended cut-off values for preoperative fibrinogen, neutrophil-lymphocyte ratio (NLR), platelet-lymphocyte ratio and lymphocyte-monocyte ratio were determined using receiver operating characteristic curve analyses. The associations between preoperative fibrinogen or D-dimer levels and clinicopathological variables were analysed using the $\chi^{2}$ test. Univariate Kaplan-Meier analysis and a multivariate Cox proportional hazards model were applied to identify which prognostic variables were significantly associated with overall survival (OS) rates. Multivariate analyses revealed that lymph node metastasis $(\mathrm{P}<0.001)$, preoperative fibrinogen $(\mathrm{P}=0.024)$ and NLR $(\mathrm{P}=0.028)$ were effective independent prognostic variables associated with OS. Based on this result, a novel, single inflammation-based combination of fibrinogen and NLR (COF-NLR) score was proposed for the determination of prognosis. Patients with elevated fibrinogen and NLR levels were allocated a score of $2(n=136)$, and those that demonstrated elevated levels of one or neither were allocated a score of $1(n=152)$ or $0(n=168)$, respectively. The 5-year OS rates were significantly poorer for patients with $C O F-N L R=2$
\end{abstract}

Correspondence to: Dr Hua-Gang Liang or Dr Kun Gao, Department of Thoracic Surgery, The First Hospital of Qinhuangdao, 258 Wenhua Road, Qinhuangdao, Hebei 066000, P.R. China

E-mail: lianghua_gang@163.com

E-mail: 15133526006@163.com

Abbreviations: OS, overall survival; NSCLC, non-small cell lung cancer; NLR, neutrophil-lymphocyte ratio; PLR, platelet-lymphocyte ratio; LMR, lymphocyte-monocyte ratio; TNM, Tumour-Node-Metastasis; COF-NLR, combination of fibrinogen and NLR

Key words: non-small cell lung cancer, fibrinogen, neutrophillymphocyte ratio, prognosis compared with those with COF-NLR $=1$ or $0(23.5 \%$ vs. $34.2 \%$ vs. $50.0 \%, \mathrm{P}<0.001)$. A subgroup analysis demonstrated that the prognostic significance of COF-NLR was independent of histological subtype, lymph node metastasis and pathological stage. Therefore, COF-NLR has potential as a novel and useful blood marker for predicting tumour progression and the postoperative survival of patients with NSCLC. It may assist clinicians in risk stratification, prognosis predictions and facilitating individualised treatment.

\section{Introduction}

Lung cancer is a leading cause of cancer-associated mortality worldwide and $80 \%$ of all lung cancer cases are non-small cell lung cancer (NSCLC) (1). Radical surgery is the standard treatment for NSCLC. However, a large number of patients experience disease progression, and therefore, a poor prognosis, within a short time following resection (2). Although several studies have been performed, the optimal prognostic factors for facilitating individualized treatments and an improvement in the prognosis of patients with NSCLC remain controversial (3). Therefore, it is of great importance to identify reliable preoperative prognostic variables to perform prognostic risk stratification and provide individualised treatment.

Previously, significant attention has been paid to the association between malignancies and coagulation (4). Among the coagulation factors, fibrinogen and D-dimer have been widely studied and are regarded as important prognostic factors in multiple types of solid tumour; including colorectal (5), uterine (6), cervical (7) and breast carcinoma (8). However, data regarding the prognostic significance of fibrinogen and D-dimer levels in NSCLC are lacking.

It has been widely recognized that cancer-related inflammatory variables, including the neutrophil-lymphocyte ratio (NLR), platelet-lymphocyte ratio (PLR) and lymphocyte-monocyte ratio (LMR), serve an important role in the promotion of cell proliferation, angiogenesis and tumour metastasis, and in the inhibition of apoptosis $(9,10)$. Recently, an increasing number of studies have evaluated the prognostic significance of the combination of fibrinogen and NLR (COF-NLR) to predict postoperative survival in patients with oesophageal squamous cell and colon cancer (11-13). However, the prognostic significance of these two variables in patients with NSCLC has not been fully investigated. 
Therefore, in the present study, a retrospective analysis was conducted to assess the prognostic relevance of preoperative coagulation factors and inflammatory indicators in a cohort of patients with non-metastatic NSCLC. Furthermore, a novel prognostic score was established by combining the optimal coagulation and inflammatory indicator status to assess the prognosis of patients with NSCLC that have undergone a complete resection.

\section{Patients and methods}

Patient selection. A total of 456 histopathologically-confirmed patients with NSCLC, who had undergone complete pulmonary resection and systematic lymphadenectomy with curative intent at the Department of Thoracic Surgery, the First Hospital of Qinhuangdao between January 2007 and June 2011, were retrospectively analysed. All patients provided written informed consent for the use of their information from the hospital database in the present study.

The eligibility criteria for the present study were as follows: Histopathologically-confirmed primary NSCLC without distant metastasis, complete surgical resection and systematic node dissection, no clinical evidence of infection or other inflammatory conditions, no autoimmune or coagulation disorders, no preoperative chemotherapy, radiotherapy or other treatment, complete clinicopathological records, including preoperative coagulation test, blood cell counts and complete follow-up as described below.

The preoperative evaluation included a physical examination, biochemical tests, coagulation tests, complete blood cell counts, flexible bronchoscopy, chest radiography, computed tomography (CT) scans, brain magnetic resonance imaging and radionuclide bone scans. The clinical stage was evaluated on the basis of the 7th edition of the Tumour-Node-Metastasis (TNM) classification for NSCLC by the Union for International Cancer Control (14).

Coagulation measurements and blood cell count analysis. Fasting preoperative venous blood samples were obtained within one week prior to surgery and were stored in a blood collection tube containing EDTA. Preoperative plasma fibrinogen concentrations were assayed based on the Clauss clotting method using DADE Thrombin Reagent ${ }^{\mathrm{TM}}$ and a Sysmex CA-7000 analyser (Sysmex Corporation, Kobe, Japan) (15). The plasma D-dimer level was measured by Microparticle Enzyme-linked Immunoassay using AxSYM analyser (Abbott Laboratories, Chicago, IL, USA), according to the manufacturer's protocols.

Complete blood cell counts and differential white blood cell count analysis of all patients were performed using the Sysmex XE-5000 automated haematology analyser (cat. no., M349228; Sysmex Corporation, Kobe, Japan). The NLR was defined as the neutrophil count/the lymphocyte count. The PLR was defined as the platelet count/the lymphocyte count. The LMR was defined as the absolute lymphocyte count/the absolute monocyte count.

Definition of COF-NLR score. The preoperative COF-NLR score was calculated by combining the fibrinogen and NLR, as previously described (11-13); patients with elevated plasma fibrinogen and NLR levels were assigned a score of 2, those that demonstrated elevated levels of one or neither were assigned a score of 1 and 0 , respectively.

Follow-up assessments. Following surgery, all patients received a follow-up evaluation via verbal or written correspondence or hospital visits every 3 months for the first year, every 6 months for the second year and subsequently every year until mortality or the final follow-up date (June 2016). Physical and laboratory examinations, as well as imaging studies, were performed at each hospital visit. Patient check-up included physical examination, tumor-marker examination, chest radiography, CT scans, neck and abdominal ultrasonography, and radionuclide bone scans when necessary to detect recurrence and/or metastasis. Overall survival (OS) time was calculated as the period from the date of surgery to the date of the patient's mortality or the final follow-up. The follow-up rate was $95.6 \%$ and the overall median follow-up time of the entire cohort was 42 months (range, 3-108 months).

Statistical analysis. All statistical analyses were performed using SPSS software version 18.0 (SPSS, Inc., Chicago, IL, USA). The most appropriate cut-off values of the preoperative fibrinogen, NLR, PLR and LMR for survival prediction were calculated using receiver operating characteristic (ROC) curve analyses. The area under the curve (AUC) was determined to estimate the diagnostic accuracy. The $\chi^{2}$ test was used to analyse statistical associations between categorical variables. The 5-year OS rate was calculated by Kaplan-Meier analysis and compared using the log-rank test. Factors identified as potentially significant by univariate analyses $(\mathrm{P}<0.05)$ were entered into a multivariate analysis with Cox regression model to identify the significant independent prognostic factors. The foundation of a novel prognostic score was based on the risk factors identified in the Cox proportional hazard model. $\mathrm{P}<0.05$ was considered to indicate a statistically significant difference.

\section{Results}

Clinicopathological variables of patients. A total of 456 patients with NSCLC with complete clinicopathological records were enrolled in the present study. The enrolled cohort consisted of $318(69.7 \%)$ males and 138 (30.3\%) females, with a median age of 61 years (range, 35-81 years). Of the enrolled patients, $387(84.9 \%)$ underwent lobectomy and $69(15.1 \%)$ underwent pneumonectomy. A total of 238 (52.2\%) patients exhibited squamous cell carcinoma, 157 (34.4\%) patients exhibited adenocarcinoma and 61 (13.4\%) patients exhibited an unspecified type of post-operative pathological subtype. A total of 170 patients presented with stage I TNM, 101 with stage II and 185 with stage IIIA.

Determination of the appropriate cut-off values for fibrinogen, NLR, PLR, LMR and D-dimer levels. The median value of plasma fibrinogen concentrations was $3.61 \mathrm{~g} / \mathrm{l}$. The optimal fibrinogen cut-off point was $3.77 \mathrm{~g} / 1$, according to the ROC analysis, with an AUC of 0.635 ( $\mathrm{P}<0.001$; Fig. 1A). The median value of NLR was 2.21. The optimal cut-off value was 2.28, according to the ROC analysis, with an AUC 

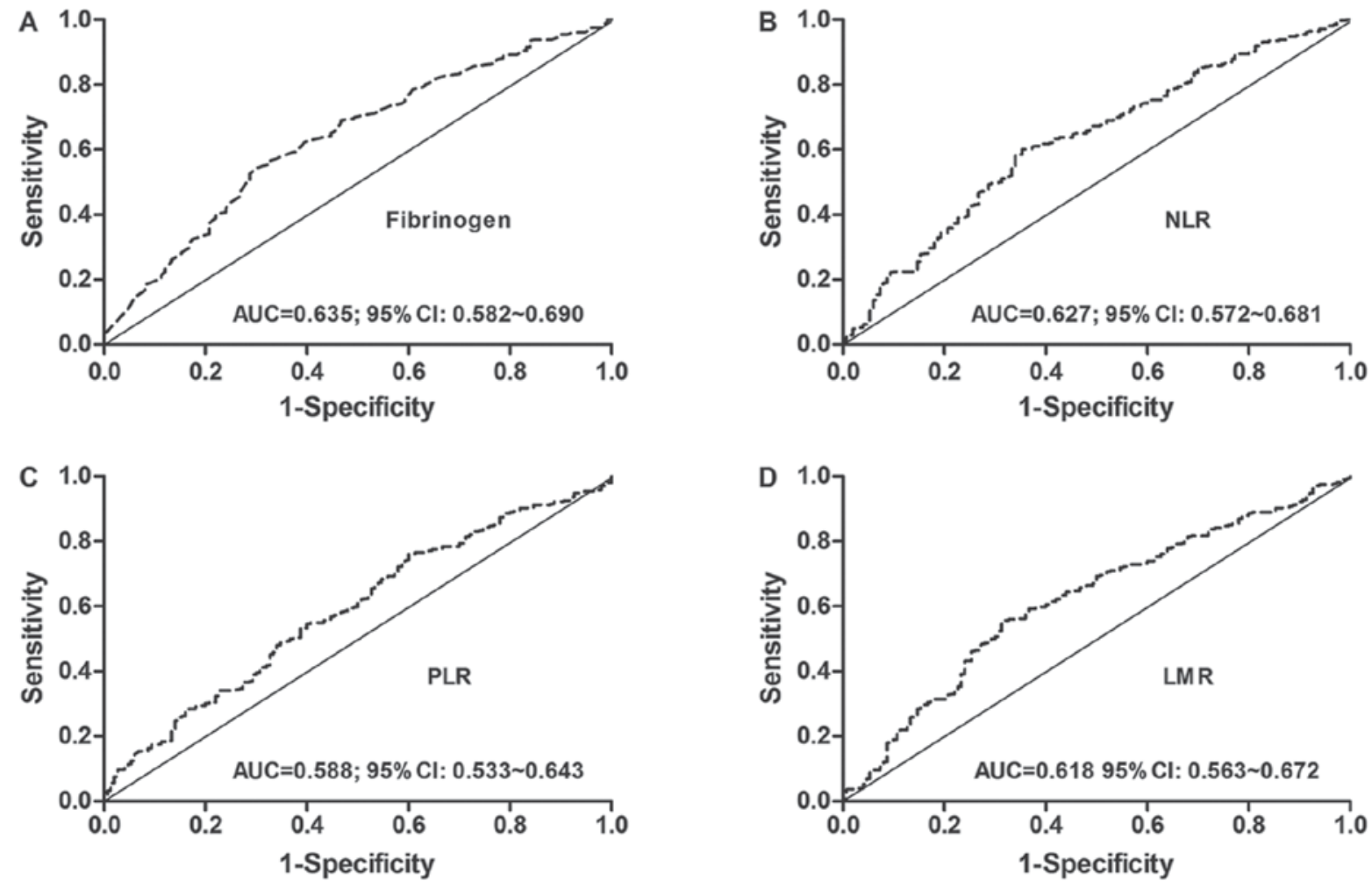

Figure 1. Receiver operating characteristic curve analyses for the cut-off values of (A) fibrinogen, (B) NLR, (C) PLR and (D) LMR in patients with NSCLC. NLR, neutrophil-lymphocyte ratio; PLR, platelet-lymphocyte ratio; LMR, lymphocyte-monocyte ratio; AUC, area under the curve; CI, confidence interval; NSCLC, non-small cell lung cancer. ROC analysis was used to calculate P-values.

of $0.627(\mathrm{P}<0.001$; Fig. 1B). The median value of PLR was 125.45. The optimal cut-off point was 106.24 , according to the ROC analysis, with an AUC of 0.588 ( $\mathrm{P}<0.001$; Fig. 1C). The median value of LMR was 3.62. The optimal cut-point was 3.70, according to the ROC analysis, with an AUC of 0.618 $(\mathrm{P}<0.001$; Fig. 1D).

Based on these cut-off point values, patients were divided into two groups for each measured variable: Fibrinogen [low ( $\leq 3.77 \mathrm{~g} / \mathrm{l}, \mathrm{n}=246)$ and high $(>3.77 \mathrm{~g} / \mathrm{l}, \mathrm{n}=210)$ ], NLR [low $(\leq 2.28, \mathrm{n}=242)$ and high $(>2.28, \mathrm{n}=214)]$, PLR [low $(\leq 106.24$, $\mathrm{n}=145)$ and high $(>106.24, \mathrm{n}=311)]$, and LMR [low $(\leq 3.7$, $\mathrm{n}=240)$ and high $(>3.7, \mathrm{n}=216)]$.

Furthermore, the reference value for D-dimer was $\leq 500 \mathrm{ng} / \mathrm{ml}$. Among the enrolled patients, 325 (71.3\%) demonstrated low plasma D-dimer levels (D-dimer $\leq 500 \mathrm{ng} / \mathrm{ml}$ ) and 131 (28.7\%) demonstrated high plasma D-dimer levels (D-dimer $>500 \mathrm{ng} / \mathrm{ml}$ ). Patients were therefore categorized into two groups according to plasma D-dimer levels: Low (D-dimer $\leq 500 \mathrm{ng} / \mathrm{ml}$ ) and high (D-dimer $>500 \mathrm{ng} / \mathrm{ml}$ ).

Association between preoperative fibrinogen or D-dimer levels and clinicopathological variables. The associations between preoperative plasma fibrinogen or D-dimer levels and the clinicopathological variables of patients with NSCLC are presented in Table I. These results revealed that high preoperative plasma fibrinogen levels were significantly associated with sex $(\mathrm{P}<0.001)$, age $(\mathrm{P}=0.018)$, smoking history $(\mathrm{P}<0.001)$, lesion type $(\mathrm{P}=0.001)$, $\mathrm{T}$ stage $(\mathrm{P}<0.001)$, pathological stage $(\mathrm{P}=0.002)$, NLR $(\mathrm{P}<0.001), \mathrm{PLR}(\mathrm{P}<0.001)$ and LMR $(\mathrm{P}<0.001)$. However, no statistically significant associations were identified between preoperative plasma fibrinogen level and tumour location or lymph node metastasis $(\mathrm{P}>0.05)$.

There were also significant associations between $\mathrm{D}$-dimer level and age $(\mathrm{P}=0.035)$, $\mathrm{T}$ stage $(\mathrm{P}=0.011)$, pathological stage $(\mathrm{P}=0.001)$ and NLR $(\mathrm{P}=0.005)$. However, no significant associations were identified between $\mathrm{D}$-dimer level and sex, smoking history, tumour location, lesion type, lymph node metastasis, PLR or LMR (all P>0.05; Table I).

Prognostic significance of preoperative coagulation and inflammatory indicators. Kaplan-Meier analyses and log-rank tests were performed to determine the prognostic value of coagulation factors and inflammatory indicators in predicting patient 5-year OS rates. The data demonstrated that the 5-year OS rate and median OS time of the fibrinogen-low group were significantly higher than those of the fibrinogen-high group [ 43.5 vs. $29.0 \%$ (48.0 vs. 26.0 months), $\mathrm{P}<0.001$; Fig. $2 \mathrm{~A}]$. The 5 -year OS rate and median OS time of the D-dimer-low group was significantly higher than those of the D-dimer-high group [40.3 vs. $28.2 \%$ (41.0 vs. 28.0 months), $\mathrm{P}=0.004$; Fig. $2 \mathrm{~B}$ ]. The 5 -year OS rate and median OS time of the NLR-low group were significantly higher than those of the NLR-high group [43.4 vs. $29.4 \%$ (47.0 vs 28.0 months), $\mathrm{P}<0.001$; Fig. $2 \mathrm{C}$ ]. The 5 -year OS rate and median OS time of the PLR-low group were significantly higher than those of the PLR-high group [44.1 vs. $33.4 \%$ (41.0 vs. 35.0 months), $\mathrm{P}=0.019$; Fig. 2D]. In addition, the 5-year OS probability and median OS time of the LMR-high group were also significantly higher than those of the LMR-low group [41.2 vs. $32.9 \%$ (43.0 vs. 30.0 months), $\mathrm{P}=0.003$; Fig. 2E]. 
Table I. The association of preoperative plasma fibrinogen and D-dimer levels with clinicopathological variables in patients with NSCLC.

\begin{tabular}{|c|c|c|c|c|c|c|c|c|c|}
\hline \multirow{2}{*}{$\begin{array}{l}\text { Clinicopathological } \\
\text { variable }\end{array}$} & \multirow[b]{2}{*}{$\mathrm{n}$} & \multicolumn{2}{|c|}{ Fibrinogen } & \multirow[b]{2}{*}{$\chi^{2}$} & \multirow[b]{2}{*}{ P-value } & \multicolumn{2}{|c|}{ D-dimer } & \multirow[b]{2}{*}{$\chi^{2}$} & \multirow[b]{2}{*}{ P-value } \\
\hline & & Low (\%) & High (\%) & & & Low $(\%)$ & $\operatorname{High}(\%)$ & & \\
\hline Sex & & & & 21.273 & $<0.001$ & & & 0.355 & 0.551 \\
\hline Male & 318 & 149 (46.9) & $169(53.1)$ & & & $224(70.4)$ & $94(29.6)$ & & \\
\hline Female & 138 & $97(70.3)$ & $41(29.7)$ & & & $101(73.2)$ & $37(26.8)$ & & \\
\hline Age (years) & & & & 5.552 & 0.018 & & & 4.468 & 0.035 \\
\hline$<61$ & 227 & $135(59.5)$ & $92(40.5)$ & & & $172(75.8)$ & $55(24.2)$ & & \\
\hline$\geq 61$ & 229 & $111(48.5)$ & $118(51.5)$ & & & $153(66.8)$ & $76(33.2)$ & & \\
\hline Smoking history & & & & 23.999 & $<0.001$ & & & 0.552 & 0.457 \\
\hline None & 151 & $106(70.2)$ & $45(29.8)$ & & & $111(73.5)$ & $40(26.5)$ & & \\
\hline Yes & 305 & $140(45.9)$ & $165(54.1)$ & & & $214(70.2)$ & $91(29.8)$ & & \\
\hline Tumor location & & & & 0.241 & 0.624 & & & 0.355 & 0.551 \\
\hline Left & 192 & $101(52.6)$ & $91(47.4)$ & & & $134(69.8)$ & $58(30.2)$ & & \\
\hline Right & 264 & $145(54.9)$ & $119(45.1)$ & & & $191(72.3)$ & $73(27.7)$ & & \\
\hline Lesion type & & & & 10.666 & 0.001 & & & 2.816 & 0.093 \\
\hline Peripheral & 315 & $186(59.0)$ & $129(41.0)$ & & & $232(73.7)$ & $83(26.3)$ & & \\
\hline Central & 141 & $60(42.6)$ & $81(57.4)$ & & & $93(66.0)$ & $48(34.0)$ & & \\
\hline T stage & & & & 41.301 & $<0.001$ & & & 8.992 & 0.011 \\
\hline $\mathrm{T} 1$ & 150 & $111(74.0)$ & $39(26.0)$ & & & $115(76.7)$ & $35(23.3)$ & & \\
\hline $\mathrm{T} 2$ & 258 & $121(46.9)$ & $137(53.1)$ & & & $184(71.3)$ & $74(28.7)$ & & \\
\hline $\mathrm{T} 3-4$ & 48 & $14(29.2)$ & $34(70.8)$ & & & $26(54.2)$ & $22(45.8)$ & & \\
\hline Lymph node metastasis & & & & 0.120 & 0.729 & & & 2.507 & 0.113 \\
\hline No & 232 & $127(54.7)$ & $105(45.3)$ & & & $173(74.6)$ & $59(25.4)$ & & \\
\hline Yes & 224 & $119(53.1)$ & $105(46.9)$ & & & $152(67.9)$ & $72(32.1)$ & & \\
\hline Pathological stage & & & & 12.467 & 0.002 & & & 13.297 & 0.001 \\
\hline I & 170 & $109(64.1)$ & $61(35.9)$ & & & $138(81.2)$ & $32(18.8)$ & & \\
\hline II & 101 & $44(43.6)$ & $57(56.4)$ & & & $64(63.4)$ & $37(36.6)$ & & \\
\hline IIIA & 185 & $93(50.3)$ & $92(49.7)$ & & & $123(66.5)$ & $62(33.5)$ & & \\
\hline NLR & & & & 49.700 & $<0.001$ & & & 7.863 & 0.005 \\
\hline Low & 242 & $168(69.4)$ & $74(30.6)$ & & & $186(76.9)$ & $56(23.1)$ & & \\
\hline High & 214 & $78(36.4)$ & $136(63.6)$ & & & $139(65.0)$ & $75(35.0)$ & & \\
\hline PLR & & & & 19.301 & $<0.001$ & & & 2.895 & 0.089 \\
\hline Low & 145 & $100(69.0)$ & $45(31.0)$ & & & 111 (76.6) & $34(23.4)$ & & \\
\hline High & 311 & 146 (46.9) & $165(53.1)$ & & & $214(68.8)$ & $97(31.2)$ & & \\
\hline LMR & & & & 30.757 & $<0.001$ & & & 0.706 & 0.401 \\
\hline Low & 240 & 100 (41.7) & $140(58.3)$ & & & 167 (69.6) & $73(30.4)$ & & \\
\hline High & 216 & $146(67.6)$ & $70(32.4)$ & & & $158(73.1)$ & $58(26.9)$ & & \\
\hline
\end{tabular}

NLR, neutrophil-lymphocyte ratio; PLR, platelet-lymphocyte ratio; LMR, lymphocyte-monocyte ratio. $\chi^{2}$ test was used to calculate the P-values.

Univariate and multivariate survival analyses of independent prognostic variables in patients with NSCLC. To further identify predictors of postoperative OS, clinicopathological variables, including coagulation and inflammatory indicators, were evaluated using univariate and multivariate analyses. Univariate analyses for OS with a log-rank test demonstrated that smoking history $(\mathrm{P}=0.023)$, lesion type $(\mathrm{P}=0.030)$, resection type $(\mathrm{P}=0.018)$, $\mathrm{T}$ stage $(\mathrm{P}=0.025)$, lymph node metastasis
$(\mathrm{P}<0.001)$, plasma fibrinogen $(\mathrm{P}<0.001)$, $\mathrm{D}$-dimer $(\mathrm{P}=0.004)$, NLR $(\mathrm{P}<0.001)$, PLR $(\mathrm{P}=0.019)$ and LMR $(\mathrm{P}=0.003)$ were significantly associated with patient prognosis (Table II).

All 10 factors indicated as significant by univariate analyses were further subjected to multivariate analyses with a Cox proportional hazards model to determine the independent prognostic factors associated with OS. The results indicated that lymph node metastasis $(\mathrm{P}<0.001)$, plasma fibrinogen level 

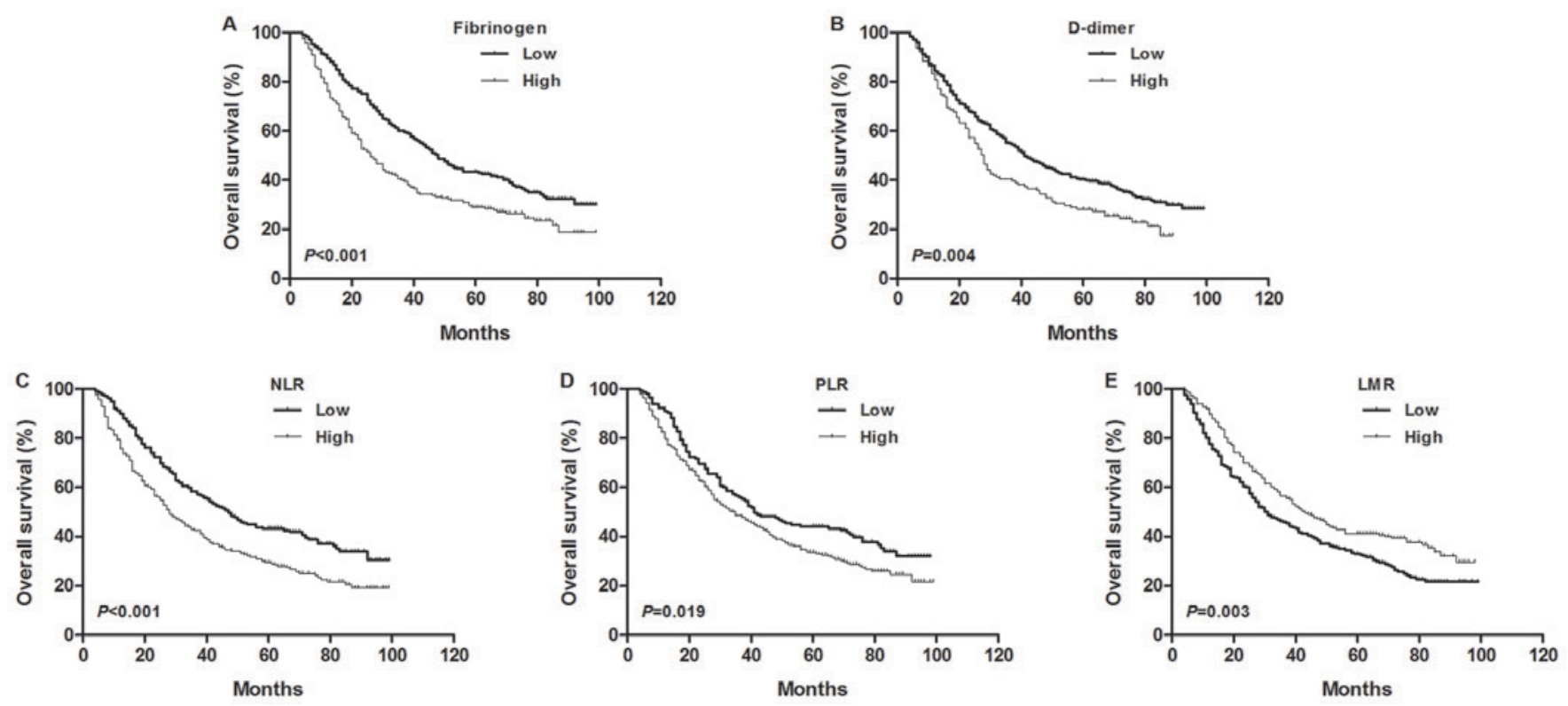

Figure 2. Kaplan-Meier survival curves for OS according to preoperative (A) fibrinogen, (B) D-dimer, (C) NLR, (D) PLR and (E) LMR in patients with NSCLC. OS, overall survival; NLR, neutrophil-lymphocyte ratio; PLR, platelet-lymphocyte ratio; LMR, lymphocyte-monocyte ratio; COF-NLR, combination of fibrinogen and NLR; NSCLC, non-small-cell lung cancer. Kaplan-Meier survival analysis with the log-rank test was used to calculate P-values.

$(\mathrm{P}=0.024)$ and NLR $(\mathrm{P}=0.028)$ were significant independent prognostic variables associated for a poor OS rate (Table III).

Prognostic analysis based on COF-NLR score for NSCLC patients. According to the grading system of the COF-NLR score, $168(36.8 \%)$ patients had a COF-NLR score of 0,152 (33.3\%) patients had a COF-NLR score of 1 and $136(29.8 \%)$ patients had a COF-NLR score of 2. The 5-year OS rates for patients with COF-NLR scores of 0,1 and 2 were 50.0, 34.2 and $23.5 \%$, respectively $(\mathrm{P}<0.001$; Fig. 3 ). Therefore, it was possible to classify the patients into three distinct prognostic groups using the preoperative COF-NLR score.

Prognostic significance of preoperative COF-NLR in subgroups of patients. The results of the present study demonstrated that preoperative COF-NLR was significantly associated with OS. To further evaluate the prognostic value of COF-NLR in subgroups of patients with NSCLC, the patients were further classified according to histopathological subtype, lymph node status and pathological stage. The results revealed that, in the squamous cell cancer subgroup, COF-NLR exhibited notable prognostic value in predicting poorer OS rates (Fig. 4A) and was also effective in patients with adenocarcinoma (Fig. 4B). In addition, COF-NLR was a prognostic indicator for OS in patients with or without lymph node metastasis (Fig. 5). Furthermore, when stratifying the patients based on clinical stage, COF-NLR also retained its predictive value for patients with pathological stage I, II or IIIA tumours (Fig. 6). Therefore, it appears that COF-NLR may serve as a powerful prognostic factor for categorising patients with NSCLC into different risk subgroups.

\section{Discussion}

Establishing one optimal preoperative prognostic factor for facilitating individual risk stratification and management is critical for improving outcomes for patients with NSCLC. Although numerous studies have been conducted to search for predictors, there is a continuing debate regarding the optimal preoperative prognostic factor for NSCLC (16-18). The activated coagulation system and systematic inflammatory response serve important functions in tumour invasion, angiogenesis promotion, metastasis and poor prognosis in several types of malignant disease $(3,19,20)$. Therefore, the present study evaluated the clinical and prognostic value of preoperative coagulation factor levels (fibrinogen and D-dimer) and inflammatory variables (NLR, PLR and LMR), and assessed the prognostic value of combining fibrinogen with NLR in NSCLC as a novel, single prognostic model.

Tumour-mediated activation of the coagulation pathway is associated with tumour growth, angiogenesis promotion and metastasis (21). Fibrinogen and D-dimer are key components of the coagulation system. Fibrinogen, an acute-phase reactant glycoprotein, is involved in the thrombin transformation of the coagulation cascade and clot formation (22). D-dimer, a stable end product of fibrin degradation, is associated with the presence of venous thromboembolism in cancer patients (23). The present study analysed the association between preoperative fibrinogen, D-dimer and clinicopathological variables. It identified that elevated fibrinogen was associated with the tumour size (T) and TNM stages, which is consistent with the findings of Zeng et al (3). Furthermore, the association between preoperative elevated D-dimer levels and clinicopathological variables in NSCLC was reported for the first time, and the results demonstrated that elevated D-dimer levels were also associated with the T and TNM stages. In addition, the results of multivariate prognostic analyses revealed that preoperative elevated fibrinogen levels, not D-dimer levels, was an independent prognostic factor for patients with NSCLC. However, the mechanism for this effect remains to be elucidated. A previous study demonstrated that interleukin- 6 produced by 
Table II. Univariate survival analysis of prognostic factors by the Kaplan-Meier method in patients with NSCLC.

\begin{tabular}{|c|c|c|c|c|c|}
\hline Clinicopathological variable & $\mathrm{n}$ & 5 -year OS rate, $\%$ & MST & $\chi^{2}$ value & P-value \\
\hline Sex & & & & 0.782 & 0.377 \\
\hline Male & 318 & 36.5 & 36.0 & & \\
\hline Female & 138 & 37.7 & 39.0 & & \\
\hline Age, years & & & & 1.404 & 0.236 \\
\hline$<61$ & 227 & 37.4 & 40.0 & & \\
\hline$\geq 61$ & 229 & 36.2 & 33.0 & & \\
\hline Smoking history & & & & 5.155 & 0.023 \\
\hline None & 151 & 43.0 & 44.0 & & \\
\hline Yes & 305 & 33.8 & 33.0 & & \\
\hline Histological type & & & & 0.528 & 0.768 \\
\hline Squamous & 238 & 35.7 & 35.0 & & \\
\hline Adenocarcinoma & 157 & 37.6 & 39.0 & & \\
\hline Others & 61 & 39.3 & 32.0 & & \\
\hline Tumor location & & & & 1.418 & 0.234 \\
\hline Left & 192 & 34.4 & 34.0 & & \\
\hline Right & 264 & 38.6 & 39.0 & & \\
\hline Lesion type & & & & 4.735 & 0.030 \\
\hline Peripheral & 315 & 39.7 & 41.0 & & \\
\hline Central & 141 & 30.5 & 32.0 & & \\
\hline Resection type & & & & 5.592 & 0.018 \\
\hline Lobectomy & 387 & 38.8 & 39.0 & & \\
\hline Pneumonectomy & 69 & 26.1 & 30.0 & & \\
\hline T stage & & & & 7.374 & 0.025 \\
\hline $\mathrm{T} 1$ & 150 & 39.3 & 41.0 & & \\
\hline $\mathrm{T} 2$ & 258 & 38.5 & 38.0 & & \\
\hline $\mathrm{T} 3-4$ & 48 & 20.8 & 25.0 & & \\
\hline Lymph node metastasis & & & & 46.357 & $<0.001$ \\
\hline No & 232 & 50.0 & 58.0 & & \\
\hline Yes & 224 & 23.2 & 27.0 & & \\
\hline Fibrinogen level & & & & 15.413 & $<0.001$ \\
\hline Low & 246 & 43.5 & 48.0 & & \\
\hline High & 210 & 29.0 & 26.0 & & \\
\hline D-dimer & & & & 8.072 & 0.004 \\
\hline Low & 325 & 40.3 & 41.0 & & \\
\hline High & 131 & 28.2 & 28.0 & & \\
\hline NLR & & & & 15.979 & $<0.001$ \\
\hline Low & 242 & 43.4 & 47.0 & & \\
\hline High & 214 & 29.4 & 28.0 & & \\
\hline PLR & & & & 5.503 & 0.019 \\
\hline Low & 145 & 44.1 & 41.0 & & \\
\hline High & 311 & 33.4 & 35.0 & & \\
\hline LMR & & & & 8.967 & 0.003 \\
\hline Low & 240 & 32.9 & 30.0 & & \\
\hline High & 216 & 41.2 & 43.0 & & \\
\hline
\end{tabular}

OS, overall survival; MST, median survival time; NLR, neutrophil-lymphocyte ratio; PLR, platelet-lymphocyte ratio; LMR, lymphocyte-monocyte ratio. Kaplan-Meier survival analysis with the log-rank test was used to calculate P-values.

cancer cells could stimulate the secretion of fibrinogen in lung cancer (24). Furthermore, elevated fibrinogen can promote angiogenesis and facilitate tumour cell metastasis by serving as an extracellular matrix for migration $(25,26)$. 
Table III. Multivariate survival analysis of independent prognostic factors by the Cox proportional hazard model in patients with NSCLC.

\begin{tabular}{|c|c|c|c|c|c|c|}
\hline Clinicopathological variable & $\mathrm{B}$ & SE & Wald & P-value & HR & $95 \% \mathrm{CI}$ \\
\hline Smoking history & -0.066 & 0.132 & 0.249 & 0.618 & 0.936 & $0.722 \sim 1.214$ \\
\hline Lesion type & -0.087 & 0.135 & 0.412 & 0.521 & 0.917 & $0.703 \sim 1.195$ \\
\hline Resection type & 0.138 & 0.163 & 0.717 & 0.397 & 1.148 & $0.834 \sim 1.579$ \\
\hline T stage & 0.033 & 0.095 & 0.119 & 0.730 & 1.033 & $0.858 \sim 1.244$ \\
\hline Lymph node metastasis & 0.754 & 0.118 & 41.020 & $<0.001$ & 2.125 & $1.687 \sim 2.676$ \\
\hline Fibrinogen level & 0.299 & 0.132 & 5.119 & 0.024 & 1.348 & $1.041 \sim 1.746$ \\
\hline D-dimer & 0.238 & 0.123 & 3.775 & 0.058 & 1.269 & $0.998 \sim 1.614$ \\
\hline NLR & 0.295 & 0.134 & 4.858 & 0.028 & 1.344 & $1.033 \sim 1.747$ \\
\hline PLR & 0.044 & 0.132 & 0.112 & 0.738 & 1.045 & $0.806 \sim 1.355$ \\
\hline LMR & -0.085 & 0.128 & 0.437 & 0.509 & 0.919 & $0.715 \sim 1.181$ \\
\hline
\end{tabular}

NLR, neutrophil-lymphocyte ratio; PLR, platelet-lymphocyte ratio; LMR, lymphocyte-monocyte ratio; HR, hazard ratio; CI, confidence interval. Multivariate survival analysis with Cox regression model was used to calculate P-values.

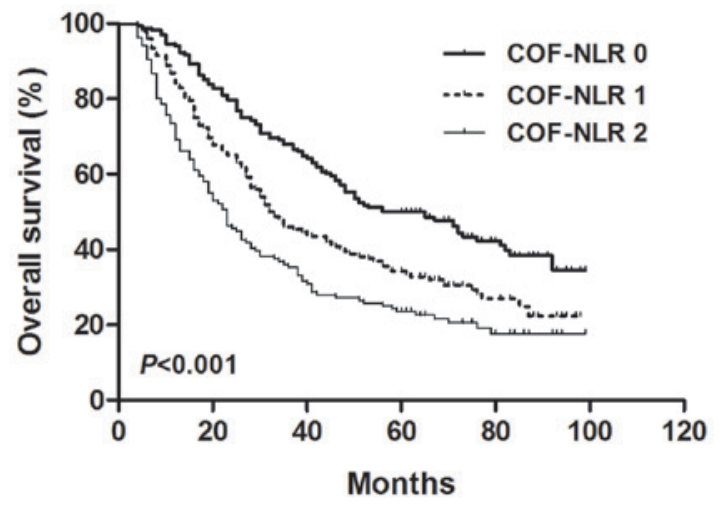

Figure 3. Kaplan-Meier survival curve for overall survival according to the preoperative COF-NLR score in patients with NSCLC. COF-NLR, combination of fibrinogen and NLR; NSCLC, non-small cell lung cancer. Kaplan-Meier survival analysis with the log-rank test was used to calculate P-values.

Imbalance in the host systematic inflammatory system serves an important role in tumour progression, proliferation and metastasis (27). A systematic inflammatory response causes variations in the number of circulating white blood cells, including neutrophils, lymphocytes and monocytes, during cancer progression (11). Previous studies have reported that three haematological indices, as the ratio of absolute counts of these three white cell constituents (NLR, PLR and LMR) were independent prognostic factors in NSCLC (28), gastric cancer (29) and oesophageal cancer (30). The present study examined the prognostic value of preoperative NLR, PLR and LMR in patients with NSCLC. The results demonstrated that a decreased 5-year OS rate in patients with NSCLC was associated with a higher NLR and PLR, and a lower LMR. In multivariate survival analysis, a higher preoperative NLR was demonstrated to be an independent prognostic factor for patients with NSCLC. Neutrophils promote tumour angiogenesis by releasing angiogenic factors, including vascular endothelial growth factor, angiopoietin-1 and fibroblast growth factor-2 (31). Lymphocytes can induce the suppression of antitumor immunity by releasing inhibitory immunological mediators, including interleukin-10 and transforming growth factor- $\beta$ (32). This may explain why an elevated NLR was associated with poorer survival rates in patients with NSCLC.

The present study also investigated the association between the three inflammatory indictors and coagulation factors, and demonstrated that higher fibrinogen levels were associated with NLR, PLR and LMR, whereas elevated D-dimer levels were only associated with higher NLR. Host inflammatory (monocyte/macrophage) cell-mediated triggering of clotting activation may be one possible mechanism in NSCLC (33). However, the mechanism behind activation of coagulation due to an increase in host inflammatory response requires further study.

As fibrinogen and NLR were demonstrated to be independent prognostic factors for patients with NSCLC in the present study, COF-NLR may aid the identification of patients with a poor prognosis following surgery and the provision of individualised treatment. In previous studies, the combined prognostic value of fibrinogen with inflammatory indicators has been discussed for gastric (11), bladder (34), hepatocellular (13) and oesophageal squamous cell carcinoma (35). However, the prognostic significance of COF-NLR has not been reported in NSCLC and this was investigated in the present study. Based on the preoperative COF-NLR score proposed in the current study, patients with NSCLC were divided into three distinct risk groups. The prognostic value of COF-NLR for histological subtype, lymph node metastasis and pathological stage subgroups was also investigated. The COF-NLR maintained its prognostic value in OS prediction for all subgroups of patients with NSCLC, indicating the reliable prognostic value of COF-NLR. To the best of our knowledge, the present study was the first to investigate the prognostic value of COF-NLR in patients with NSCLC. Considering that fibrinogen and NLR are markers that can be readily evaluated in conventional blood analyses (11), the preoperative assessment of COF-NLR may be suitable as a reliable prognostic tool for patients with NSCLC; the lower-scoring COF-NLR groups are associated with an improved prognosis compared with the higher-scoring COF-NLR groups. 

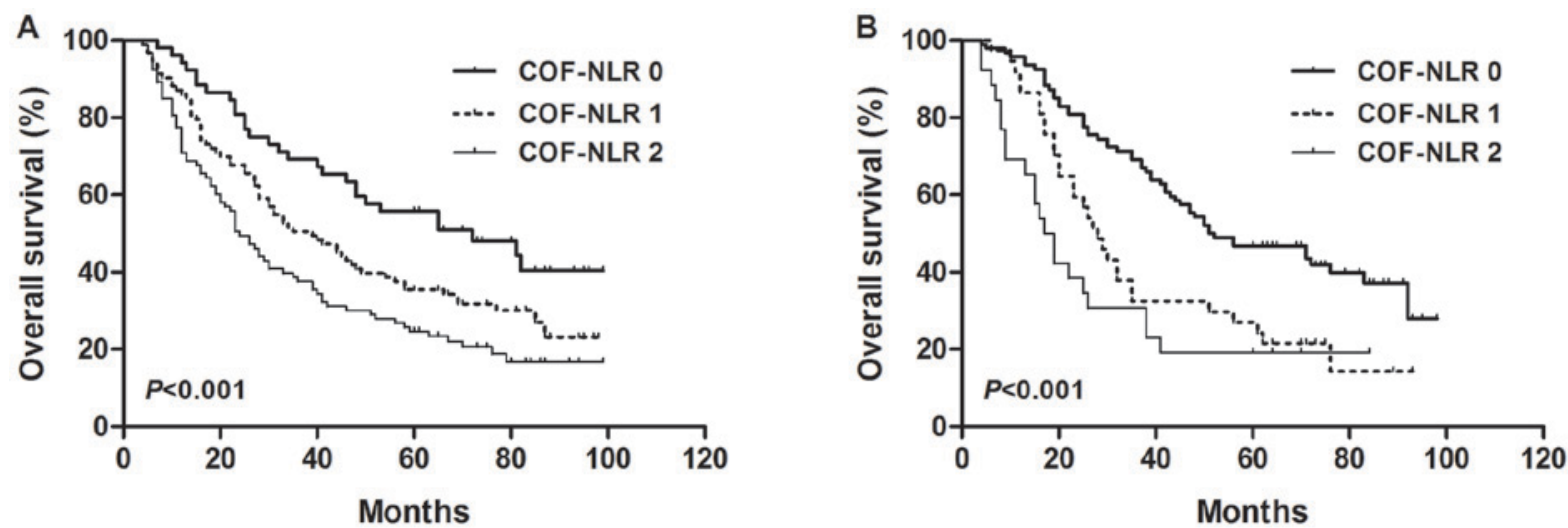

Figure 4. Kaplan-Meier survival curve for overall survival according to the COF-NLR score in (A) patients with primary squamous cell carcinoma and (B) patients with adenocarcinoma. COF-NLR, combination of fibrinogen and NLR. Kaplan-Meier survival analysis with the log-rank test was used to calculate P-values.
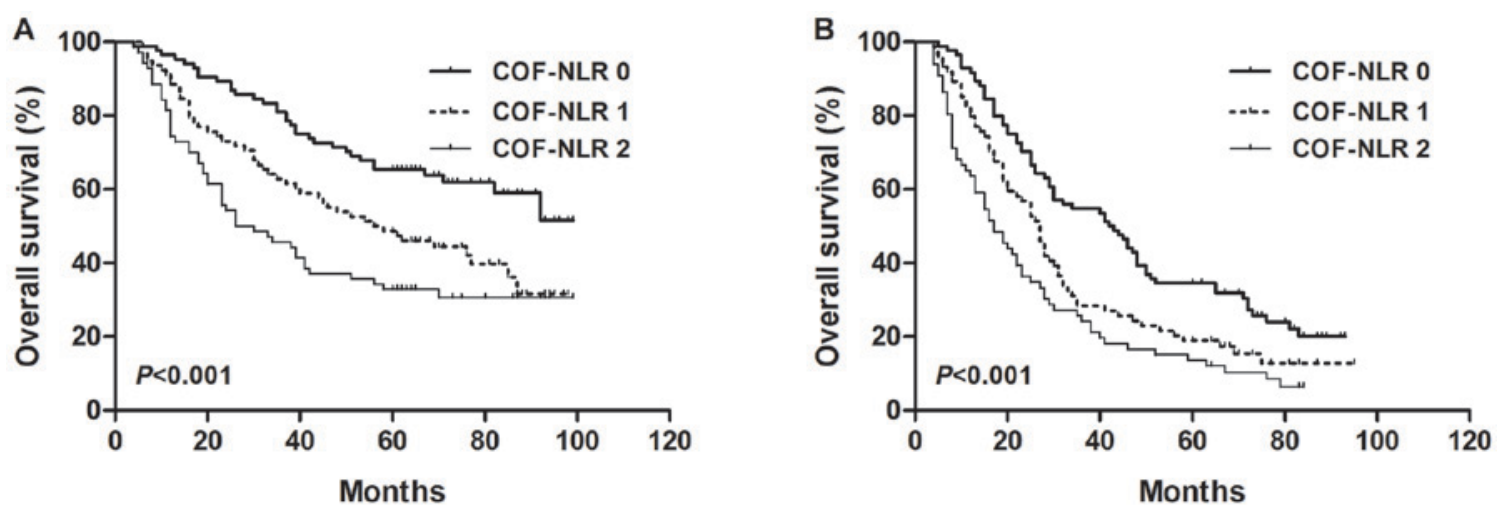

Figure 5. Kaplan-Meier survival curves for overall survival according to the COF-NLR score in (A) NO NSCLC patients and (B) N1-N3 NSCLC patients. COF-NLR, combination of fibrinogen and NLR; NSCLC, non-small cell lung cancer. Kaplan-Meier survival analysis with the log-rank test was used to calculate P-values.
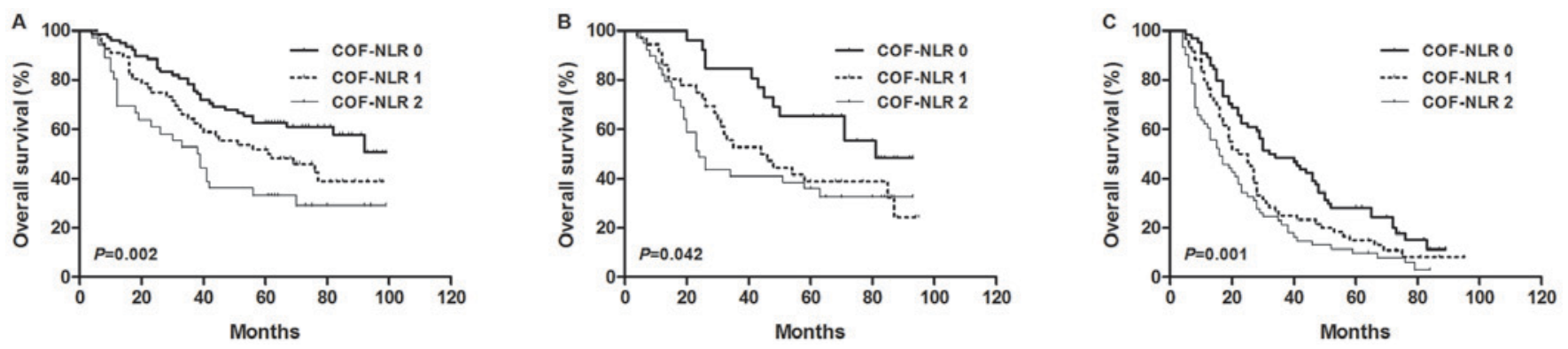

Figure 6. Kaplan-Meier survival curves for overall survival according to COF-NLR score of (A) pathological stage I NSCLC patients, (B) stage II NSCLC patients and (C) stage IIIA NSCLC patients. COF-NLR, combination of fibrinogen and NLR; NSCLC, non-small cell lung cancer. Kaplan-Meier survival analysis with the log-rank test was used to calculate P-values.

However, there are certain limitations to the present study. To begin with, this retrospective study was conducted by a single institution. Secondly, all data were selected from one relatively small sample of patients, and a control arm was not set for validation. Therefore, the preoperative prognostic significance of COF-NLR in patients with NSCLC should be further strengthened in a larger, multicentre prospective study.

In conclusion, in the present study it was identified that, of the coagulation and inflammatory indicators, preoperative fibrinogen and NLR were independent prognostic predictors for NSCLC. A novel prognostic factor was further proposed, based on the combination of fibrinogen with the NLR from blood specimen analysis (COF-NLR), and was demonstrated to have prognostic predictive potential for patients with NSCLC. In the future, by adding this prognostic factor to clinical work, it may be possible to make insightful preoperative prognostic predictions and customise individual treatment plans for patients with NSCLC. 


\section{Acknowledgements}

Not applicable.

\section{Funding}

The present study was supported by the Science Foundation of Qinhuangdao (grant no. 201502A093).

\section{Availability of data and materials}

The datasets used and/or analyzed during the present study are available from the corresponding author on reasonable request.

\section{Authors' contributions}

HL and KG performed the experiment and wrote the manuscript. HL was responsible for the design of the experiment. RJ and JL analyzed the experiment data. CW helped with the statitical analysis. JL and CW helped to revise the manuscript. All authors read and approved the final manuscript.

\section{Ethics approval and consent to participate}

The present study was approved by the Ethics Committee of the First Hospital of Qinhuangdao and written informed consent was obtained from each patient prior to enrolment.

\section{Patient consent for publication}

Each patient provided written informed consent for the publication of this study.

\section{Competing interests}

The authors declare that they have no competing interests.

\section{References}

1. Siegel RL, Miller KD and Jemal A: Cancer statistics, 2015. CA Cancer J Clin 65: 5-29, 2015.

2. Kamangar F, Dores GM and Anderson WF: Patterns of cancer incidence, mortality, and prevalence across five continents: Defining priorities to reduce cancer disparities in different geographic regions of the world. J Clin Oncol 24: 2137-2150, 2006.

3. Zeng Q, Xue N, Dai D, Xing S, He X, Li S, Du Y, Huang C, Li L and Liu W: A nomogram based on inflammatory factors C-reactive protein and fibrinogen to predict the prognostic value in patients with resected non-small cell lung cancer. JCancer 8: 744-753, 2017.

4. Murray JC: Coagulation and cancer. Br J Cancer 64: 422-424, 1991.

5. Son HJ, Park JW, Chang HJ, Kim DY, Kim BC, Kim SY, Park SC, Choi HS and Oh JH: Preoperative plasma hyperfibrinogenemia is predictive of poor prognosis in patients with nonmetastatic colon cancer. Ann Surg Oncol 20: 2908-2913, 2013.

6. Vahid DM, Ahmari S, Alipour S and Tehranian A: The comparison of plasma $\mathrm{D}$-dimer levels in benign and malignant tumors of cervix, ovary and uterus. Int J Hematol Oncol Stem Cell Res 9: 107-111, 2015.

7. Nakamura K, Nakayama K, Ishikawa M, Katagiri H, Minamoto T, Ishibashi T, Ishikawa N, Sato E, Sanuki K, Yamashita H, et al: High pre-treatment plasma D-Dimer level as a potential prognostic biomarker for cervical carcinoma. Anticancer Res 36: 2933-2938, 2016

8. Dirix LY, Salgado R, Weytjens R, Colpaert C, Benoy I, Huget P, van Dam P, Prové A, Lemmens J and Vermeulen P: Plasma fibrin D-dimer levels correlate with tumour volume, progression rate and survival in patients with metastatic breast cancer. $\mathrm{Br}$ J Cancer 86: 389-395, 2002.
9. Dunn GP, Old LJ and Schreiber RD: The immunobiology of cancer immunosurveillance and immunoediting. Immunity 21 : 137-148, 2004.

10. McMillan DC: Systemic inflammation, nutritional status and survival in patients with cancer. Curr Opin Clin Nutr Metab Care 12: 223-226, 2009

11. Arigami T, Uenosono Y, Matsushita D, Yanagita S, Uchikado Y, Kita Y, Mori S, Kijima Y,Okumura H, Maemura K, et al: Combined fibrinogen concentration and neutrophil-lymphocyte ratio as a prognostic marker of gastric cancer. Oncol Lett 11: 1537-1544, 2016.

12. Arigami T, Okumura H, Matsumoto M, Uchikado Y, Uenosono Y, Kita Y, Owaki T, Mori S, Kurahara H, Kijima Y, et al: Analysis of the fibrinogen and neutrophil-lymphocyte ratio in esophageal squamous cell carcinoma: A promising blood marker of tumor progression and prognosis. Medicine (Baltimore) 94: e1702, 2015.

13. Fu SJ, Ji F, Han M, Chen MG, Wang XP, Ju WQ, Zhao Q, Wu LW, Ren QQ, Guo ZY, et al: Prognostic value of combined preoperative fibrinogen and neutrophil-lymphocyte ratio in patients with hepatocellular carcinoma after liver transplantation. Oncotarget 8: 4301-4312, 2017.

14. Xia H, Sun Z, Deng L, Zhu D and Wang D: Prognostic significance of the preoperative lymphocyte to monocyte ratio in patients with stage I non-small cell lung cancer undergoing complete resection. Cancer Invest 34: 378-384, 2016.

15. Dorn-Beineke A, Dempfle CE, Bertsch T and Wisser H: Evaluation of the automated coagulation analyzer Sysmex CA-7000. Thromb Res 116: 171-179, 2005.

16. Tian S: Classification and survival prediction for early-stage lung adenocarcinoma and squamous cell carcinoma patients. Oncol Lett 14: 5464-5470, 2017.

17. Fang L, Sheng H, Wan D, Zhu C, Jiang R, Sun X and Feng J: Prognostic role of multidrug resistance-associated protein 1 expression and platelet count in operable non-small cell lung cancer. Oncol Lett 16: 1123-1132, 2018.

18. Zou Q, Wang T, Li B, Li G, Zhang L, Wang B and Sun S: Overexpression of circ-0067934 is associated with increased cellular proliferation and the prognosis of non-small cell lung cancer. Oncol Lett 16: 5551-5556, 2018.

19. Yamashita H, Kitayama J, Kanno N, Yatomi Y and Nagawa H: Hyperfibrinogenemia is associated with lymphatic as well as hematogenous metastasis and worse clinical outcome in T2 gastric cancer. BMC Cancer 6: 147, 2006.

20. Palumbo JS: Mechanisms linking tumor cell-associated procoagulant function to tumor dissemination. Semin Thromb Hemost 34: 154-160, 2008.

21. Lal I, Dittus K and Holmes CE: Platelets, coagulation and fibrinolysis in breast cancer progression. Breast Cancer Res 15: 207, 2013.

22. Weisel JW: Fibrinogen and fibrin. Adv Protein Chem 70: 247-299, 2005.

23. Arpaia G, Carpenedo M, Verga M, Mastrogiacomo O, Fagnani D, Lanfredini M, Milani M and Cimminiello C: D-dimer before chemotherapy might predict venous thromboembolism. Blood Coagul Fibrinolysis 20: 170-175, 2009.

24. Yamaguchi T, Yamamoto Y, Yokota S, Nakagawa M, Ito M and Ogura T: Involvement of interleukin-6 in the elevation of plasma fibrinogen levels in lung cancer patients. Jpn J Clin Oncol 28: 740-744, 1998.

25. Garcia MG, Bayo J, Bolontrade MF, Sganga L, Malvicini M, Alaniz L, Aquino JB, Fiore E, Rizzo MM, Rodriguez A, et al: Hepatocellular carcinoma cells and their fibrotic microenvironment modulate bone marrow-derived mesenchymal stromal cell migration in vitro and in vivo. Mol Pharm 8: 1538-1548, 2011.

26. Zheng S, Shen J, Jiao Y, Liu Y, Zhang C, Wei M, Hao S and Zeng X: Platelets and fibrinogen facilitate each other in protecting tumor cells from natural killer cytotoxicity. Cancer Sci 100: 859-865, 2009.

27. Vesely MD and Schreiber RD: Cancer immunoediting: Antigens, mechanisms, and implications to cancer immunotherapy. Ann N Y Acad Sci 1284: 1-5, 2013.

28. Wu G, Yao Y, Bai C, Zeng J, Shi D, Gu X, Shi X and Song Y: Combination of platelet to lymphocyte ratio and neutrophil to lymphocyte ratio is a useful prognostic factor in advanced non-small cell lung cancer patients. Thorac Cancer 6: 275-287, 2015.

29. Lee S, Oh SY, Kim SH, Lee JH, Kim MC, Kim KH and Kim HJ: Prognostic significance of neutrophil lymphocyte ratio and platelet lymphocyte ratio in advanced gastric cancer patients treated with FOLFOX chemotherapy. BMC Cancer 13: 350, 2013.

30. Hirahara N, Matsubara T, Mizota Y, Ishibashi S and Tajima Y: Prognostic value of preoperative inflammatory response biomarkers in patients with esophageal cancer who undergo a curative thoracoscopic esophagectomy. BMC Surg 16: 66, 2016. 
31. Neagoe PE, Brkovic A, Hajjar F and Sirois MG: Expression and release of angiopoietin-1 from human neutrophils: Intracellular mechanisms. Growth Factors 27: 335-344, 2009.

32. Hoffmann TK, Dworacki G, Tsukihiro T, Meidenbauer N, Gooding W, Johnson JT and Whiteside TL: Spontaneous apoptosis of circulating $\mathrm{T}$ lymphocytes in patients with head and neck cancer and its clinical importance. Clin Cancer Res 8: 2553-2562, 2002.

33. Gabazza E, Taguchi O, Yamakami T, Machishi M, Ibata $\mathrm{H}$ and Suzuki S: Correlation between increased granulocyte elastase release and activation of blood coagulation in patients with lung cancer. Cancer 72: 2134-2140, 1993.
34. Ma C, Lu B, Diao C, Zhao K, Wang X, Ma B, Lu B and Sun E: Preoperative neutrophil-lymphocyte ratio and fibrinogen level in patients distinguish between muscle-invasive bladder cancer and non-muscle-invasive bladder cancer. Onco Targets Ther 9: 4917-4922, 2016.

35. Kijima T, Arigami T, Uchikado Y, Uenosono Y, Kita Y, Owaki T, Mori S, Kurahara H, Kijima Y, Okumura H, et al: Combined fibrinogen and neutrophil-lymphocyte ratio as a prognostic marker of advanced esophageal squamous cell carcinoma. Cancer Sci 108: 193-199, 2017.

This work is licensed under a Creative Commons Attribution-NonCommercial-NoDerivatives 4.0 International (CC BY-NC-ND 4.0) License. 